$\boldsymbol{s}$ VICTORY's SHADOW 



\title{
VICTORY'S SHADOW
}

\author{
CONQUEST AND GOVERNANCE
} IN MEDIEVAL CATALONIA

THOMAS W. BARTON 
Copyright (C) 2019 by Cornell University

All rights reserved. Except for brief quotations in a review, this book, or parts thereof, must not be reproduced in any form without permission in writing from the publisher. For information, address Cornell University Press, Sage House, 512 East State Street, Ithaca, New York 14850. Visit our website at cornellpress.cornell.edu.

First published 2019 by Cornell University Press

Printed in the United States of America

Library of Congress Cataloging-in-Publication Data

Names: Barton, Thomas W., 1976- author.

Title: Victory's shadow : conquest and governance in medieval Catalonia / Thomas W. Barton.

Description: Ithaca : Cornell University Press, 2019. Includes bibliographical references and index.

Identifiers: LCCN 2018042957 (print) | LCCN 2018044225 (ebook) | ISBN 9781501736186 (pdf) | ISBN 9781501736179 (epub/mobi) | ISBN 9781501736162 | ISBN 9781501736162 (cloth: alk. paper)

Subjects: LCSH: Catalonia (Spain)—History. | Catalonia (Spain)-Religion. | Spain-History-711-1516.

Christianity and other religions-Islam-HistoryTo 1500. | Islam-Relations-ChristianityHistory-To 1500 .

Classification: LCC DP302.C65 (ebook) | LCC DP302.C65 B37 2019 (print) | DDC 946.702-dc23

LC record available at https://lccn.loc.gov/ 2018042957

Cover art: Taula d'enteixinat amb cavallers, galeres i nau d'alta borda and Taula d'enteixinat amb genets. Aragon, 14th century. (C) Museu Nacional d'Art de Catalunya, Barcelona. Used by permission. 
For Whitney 
